INPLASY

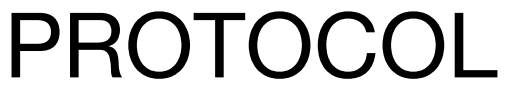

To cite: He et al. The efficacy and safety of auricular point pressing therapy for knee osteoarthritis: A protocol for systematic review and metaanalysis. Inplasy protocol

202220077. doi:

10.37766/inplasy2022.2.0077

Received: 18 February 2022

Published: 19 February 2022

Corresponding author: Jie He

sg_jiejie243@126.com

Author Affiliation:

Jiangyan hospital affiliated to Nanjing University of Chinese Medicine.

Support: None.

Review Stage at time of this submission: The review has not yet started.

Conflicts of interest: None declared.

\section{The efficacy and safety of auricular point pressing therapy for knee osteoarthritis: A protocol for systematic review and meta-analysis}

He, J1; Ma, L2; Zhou, F3; Jiang, HB².

Review question / Objective: This systematic review aims to evaluate the efficacy and safety of auricular point pressing therapy for relieving pain, stiffness, and physical dysfunction in patients with knee osteoarthritis. Is auricular point pressing therapy a safe treatment for knee osteoarthritis?

Condition being studied: Knee Osteoarthritis (KOA) is the most common degenerative joint disease of the older patients, the common symptoms of KOA are pain, stiffness of the joint, crepitation on motion and limitation of joint motion, which have a significant detrimental impact on quality of life. The main goals of treatment are relieving pain and improving function, there are several measures for the early stage of KOA including loss of weight, exercise therapy, non-steroidal anti-inflammatory drugs (NSAIDs), corticosteroid injections or glucosamine supplement. NSAIDs are the most commonly prescribed agents for pain management, however, the drug increases the risk of gastrointestinal (GI) bleeding and vascular adverse events in long-term use. Auricular acupressure (AA) was one of acupuncture-related techniques that was used as therapeutic methods in China. Limited evidence suggests that auricular point pressing therapy may be beneficial for KOA.

INPLASY registration number: This protocol was registered with the International Platform of Registered Systematic Review and Meta-Analysis Protocols (INPLASY) on 19 February 2022 and was last updated on 19 February 2022 (registration number INPLASY202220077).

\section{INTRODUCTION}

Review question / Objective: This systematic review aims to evaluate the efficacy and safety of auricular point pressing therapy for relieving pain, stiffness, and physical dysfunction in patients with knee osteoarthritis. Is auricular point pressing therapy a safe treatment for knee osteoarthritis? 
Condition being studied: Knee Osteoarthritis (KOA) is the most common degenerative joint disease of the older patients, the common symptoms of KOA are pain, stiffness of the joint, crepitation on motion and limitation of joint motion, which have a significant detrimental impact on quality of life. The main goals of treatment are relieving pain and improving function, there are several measures for the early stage of KOA including loss of weight, exercise therapy, non-steroidal antiinflammatory drugs (NSAIDs), corticosteroid injections or glucosamine supplement. NSAIDs are the most commonly prescribed agents for pain management, however, the drug increases the risk of gastrointestinal (GI) bleeding and vascular adverse events in long-term use. Auricular acupressure (AA) was one of acupuncture-related techniques that was used as therapeutic methods in China. Limited evidence suggests that auricular point pressing therapy may be beneficial for KOA.

\section{METHODS}

Participant or population: KOA patients with definite diagnosis using American College of Rheumatology (ACR) diagnostic criteria will be included.

Intervention: The treatment group will be treated with auricular point pressing therapy (there is no limit on the materials, treatment methods, and course of treatment).

Comparator: The control group was treated with placebo, drugs, or other alternative therapy. Studies comparing 2 different types of auricular point pressing therapy or surgical procedures will be expelled.

Study designs to be included: Only randomized controlled clinical trials (RCTs) related to the effects of auricular point pressing therapy for treating KOA will be included. Trials published in the form of dissertations will be selected as eligible studies.
Eligibility criteria: Designs such as animal experiments, reviews, quasi-RCTs, case reports, and laboratory studies will be rejected. The ongoing studies or unpublished data will not be included.

Information sources: Relevant studied will be searched in the following electronic databases: PubMed, EMBASE, Cochrane Library, CINAHL, China Knowledge Resource Integrated Database, Weipu Database for Chinese Technical Periodicals, Sinomed, and Wanfang Database. The search terms include auricular, point, points, knee osteoarthritis, gonarthrosis, os teoarthrosis, osteoarthropathy, arthralgia

Main outcome(s): Western Ontario and McMaster Universities Osteoarthritis Index (WOMAC) score VAS.

Quality assessment / Risk of bias analysis: We will use the Cochrane Collaboration's tool which is recommended by the Cochrane Reviewer's Handbook to assess risk of bias for quality assessment of the included studies. The studies will be graded based on: (i) random sequence generation; (ii) allocation concealment; (iii) blinding; (iv) incomplete outcome data; (v) selective outcome reporting; (vi) other sources of bias.

Strategy of data synthesis: Comparisons will be made between any form of auricular point pressing therapy and placebo or sham or no treatment with/without same additional treatment is given to both groups. The data of the study included may be divided into two cases, depending on whether the data are suitable for metaanalysis. If the meta-analysis will not be performed because of heterogeneity, interventions, comparisons, outcomes etc, we will make forms for a qualitative description. If the data is suitable for metaanalysis, we will perform the meta-analysis using software RevMan 5.3 (Review Manager). For dichotomous data, we will present the results as risk ratios (RR) with $95 \%$ confidence intervals (CIs). For continuous data, the mean difference (MD) will be included in. If outcome variables 
were measured on different scales, standard mean differences (SMD) analysis with $95 \%$ Cls will be included in the metaanalysis. For the data will be done with the meta-analysis, we should test the heterogeneity first by a standard $I^{2}$ test. If there is no statistic heterogeneity among the results, the fixed effects model will be employed for meta-analysis. If there is a statistic heterogeneity, the source of the heterogeneity should be further analyzed. If there is obvious clinical heterogeneity, the subgroup or sensitivity analysis, or only descriptive analysis can be performed.

Subgroup analysis: If there is a significant heterogeneity in the included trials, we will conduct subgroup analysis based on the severity of knee osteoarthritis and types of auricular point pressing therapy.

Sensitivity analysis: The sensitivity analysis will be conducted to test the stability of results of meta-analysis by the study of large weight of elimination effect.

Language: Without any language or publication status restrictions.

Country(ies) involved: China.

Keywords: knee osteoarthritis, auricular point pressing therapy, protocol, systematic review.

Contributions of each author:

Author 1 - Jie He - The author drafted the manuscript.

Author 2 - Lin Ma - The author provided statistical expertise.

Author 3 - Feng Zhou - The author contributed to the development of the selection criteria, and the risk of bias assessment strategy.

Author 4 - Hongbo Jiang - The author contributed to the development of the selection criteria, and the risk of bias assessment strategy. 